\title{
Risk of presentation to hospital with epileptic seizures after vaccination with monovalent AS03 adjuvanted pandemic A/H1N1 2009 influenza vaccine (Pandemrix): self controlled case series study
}

\author{
(c) (1) $(9)$ OPEN ACCESS
}

\author{
Lisen Arnheim-Dahlström research associate, Jonas Hällgren statistician, Caroline E Weibull \\ statistician, Pär Sparén professor
}

Department of Medical Epidemiology and Biostatistics, Karolinska Institutet, Nobels väg 12A, 17177 Stockholm, Sweden

\begin{abstract}
Objective To assess the risk of epileptic seizures in people with and without epilepsy after vaccination with a monovalent AS03 adjuvanted pandemic A/H1N1 influenza vaccine (Pandemrix; Glaxo SmithKline, Sweden)

Design Register based self controlled case series.

Setting Three Swedish counties (source population 750 000).

Participants 373398 people (age 0-106, median 41.2) who were vaccinated. Vaccinated people with epileptic seizures, diagnosed as inpatients or outpatients, at any time from 90 days before until 90 days after any dose of vaccine.

Main outcome measures Endpoints were admission to hospital or outpatient hospital care with epileptic seizures as the main diagnosis. The effect estimate of relative incidence was calculated as the incidence of epileptic seizures in period after exposure relative to the incidence of epileptic seizures in two control periods, one before and one after vaccination.
\end{abstract}

Results 859 people experienced epileptic seizures during the study period. There was no increased risk of seizures in people with previously diagnosed epilepsy (relative incidence 1.01, 95\% confidence interval 0.74 to 1.39 ) and a non-significant decrease in risk for people without epilepsy $(0.67,0.27$ to 1.65$)$ during the day $1-7$ risk period (where day 1 is the day of vaccination). In a second risk period (day 8-30), there was a non-significant increased risk of seizures in people without epilepsy $(1.11,0.73$ to 1.70$)$ but no increase in risk for those with epilepsy (1.00, 0.83 to 1.21$)$.

Conclusions This study found no evidence of an increase in risk of presentation to hospital with epileptic seizures after vaccination with a monovalent $\mathrm{AS} 03$ adjuvanted pandemic $\mathrm{H} 1 \mathrm{~N} 1$ influenza vaccine.

\section{Introduction}

Despite an increasing success of immunisation systems, concerns about the safety of vaccination have increased. Perceived safety issues that have been in public focus in the past decades have included disorders within the autistic spectrum ${ }^{12}$ or the neurological events after vaccination against pertussis. ${ }^{3}$ The 13th pandemic pharmacovigilance report ${ }^{4}$ by the European Medicines Agency (EMA) contains 10326 case reports received by EudraVigilance, a data processing network and management system for reporting and evaluating suspected adverse reactions during the development and after the marketing authorisation of medicinal products in the European Economic Area, after vaccination with Pandemrix (Glaxo SmithKline, Sweden), one of the four authorised pandemic vaccines. It was estimated that at least 30.4 million people were vaccinated with Pandemrix during the 2009-10 A/H1N1PDM09 pandemic. Most reported adverse events (almost 7000) were classified as general disorders and among these about 5000 cases were classified as gastrointestinal and nervous disorders. Across Europe there were 38 cases of seizures with fatal outcome in patients known to have epilepsy. Although no conclusion about a causal relation could be drawn, the reported cases merited further investigation. Vaccination against $\mathrm{H} 1 \mathrm{~N} 1$ was associated with several neurological events such as Guillain-Barré syndrome ${ }^{5}$ and has been associated with risk of narcolepsy. ${ }^{67}$

When a person has two or more unprovoked seizures, that person is considered to have epilepsy. ${ }^{8}$ In Sweden, people with severe epilepsy are advised to be vaccinated against flu as the risk of seizure and status epilepticus are increased during infection. Vaccination against H1N1 flu with Pandemrix started out in October 2009 and was offered to the general population free of charge. This is in contrast with the seasonal flu vaccine, which is free only for those aged over 65. The National Board of Health in Sweden recommended that everybody should be vaccinated against pandemic H1N1 flu and the vaccination coverage was at least $60 \%$. The yearly coverage of the seasonal flu vaccine is about $10-15 \%$ and is rare in children and in the healthy population. Since the start of the vaccination campaign, three 
counties registered all their vaccinations in a vaccination register. We assessed the risk of presenting to hospital with epileptic seizures after vaccination.

\section{Methods \\ Study population}

Three counties in Sweden-Värmland, Kalmar and Norrbotten-with a source population of 750000 used the Swedish vaccination register (SVEVAC) for registration of H1N1 vaccination. Between October 2009 and May 2010, 373 398 people in the three counties were vaccinated. It was recommended that adults would be given one dose of the vaccine and children two doses. Most vaccinations occurred during a two month period from 19 October to 20 December 2009. Outside this period there were few vaccinations.

For the analysis in this population based retrospective study, we identified two study cohorts according to recorded epilepsy from all vaccinated individuals $(n=373$ 398) identified from the vaccination register until May 2010 (fig $1 \Downarrow$ ). People who died or moved outside the counties were excluded from the study. We used regional patient registers of inpatient and outpatient hospital care in the three counties to identify individuals with primary diagnosis of epilepsy using ICD-10 (international classification of diseases, 10th revision) codes G40 and G41. We did not have access to data on visits to primary healthcare. Apart from the patient registers, however, we also used data on prescription of antiepileptic drugs (ATC $=\mathrm{N} 03 \mathrm{~A})$ from the national Swedish drug prescription register during the corresponding period. Linkage between the patient register, the prescription register, and vaccination register was possible through the unique personal identification number that is maintained for all Swedish residents. ${ }^{9}$ We created two cohorts with $(n=7787)$ and without $(n=356611)$ epilepsy, according to the diagnosis of epilepsy or prescription of antiepileptic drugs within one year before the control period before vaccination (fig $1 \Downarrow$ ). In both cohorts, we identified all admissions to hospital for epilepsy (ICD-10 codes G40 and G41) from three months (90 days) before until three months (90 days) after any dose of vaccine (fig $2 \Downarrow$ ). For an additional analysis, we also identified all admissions for febrile seizures (ICD-10 code R56). For people who received two doses of vaccine ( $n=504$ and 55447 in the cohorts with and without epilepsy, respectively), the risk and control periods were defined after each of the two doses. The risk period after dose 1 was censored at the time of dose 2 if there was a second dose less than 30 days after the first dose of vaccination.

\section{Case definition}

In the people with and without recorded epilepsy we determined how many in each cohort had at least one epileptic seizure recorded in the patient register any time from 90 days before vaccination to 90 days after to create two subcohorts: people with epilepsy and with seizures, and people without epilepsy but with seizures (fig $1 \Downarrow$ ). Only people with epileptic seizures were included in these subcohorts. In an additional analysis we used both epileptic and febrile seizures from the patient register as a composite outcome.

\section{Statistical analysis}

We calculated the incidence rates of epileptic seizures for both subcohorts as the number of seizures divided by the total number of person years. Recurrent events were assumed to be independent within individuals and therefore included in the calculations. We estimated the corresponding $95 \%$ confidence intervals using exact Poisson confidence limits. ${ }^{10}$

In the subcohorts we used the self controlled case series (SCCS) method to investigate the associations between vaccination and epileptic seizures in inpatient or outpatient hospital care. This method is based on comparisons of event rates in different periods within each individual's observation time and involves only exposed cases - that is, individuals who have experienced the outcome of interest. ${ }^{11}$ The method controls implicitly for all fixed confounders that do not vary over the observation period-for example, genetics, location, socioeconomic status, sex, individual frailty, severity of underlying disease. Fixed effects Poisson regression models were fitted to the data to estimate the relative incidence and corresponding $95 \%$ confidence intervals for the epileptic seizure cohort and the non-epileptic seizure cohort. The relative incidences were calculated as the ratio of the incidence rate of epileptic seizures in the risk period(s) to the rate of epileptic seizures in the control period(s). We defined two risk periods: day 1 to day 7 , and day 8 to day 30 after vaccination (day 1 being the day of vaccination). These risk periods were compared with a two month control period, comprising 90 to 30 days before vaccination and 31 to 90 days after vaccination (fig $2 \Downarrow$ ). We also defined a one month buffer period of 30 days to one day before vaccination as seizure rates shortly before vaccination might be artificially lower or higher. Because of the short observation period (six months) we did not include an age effect in the model. In an additional analysis we ran the same models with epileptic and febrile seizures as a composite outcome. Statistical calculations were done in SAS version 9.2, using the SAS macro developed by Whitaker et al. ${ }^{12}$

\section{Results}

The mean age at vaccination was 41 (SD 25); and this did not differ between those with and without epilepsy (data not shown). Most vaccinations in people with epilepsy were done at ages 25-74, while the vaccinations in those without epilepsy were more evenly distributed over ages, except for those over 75 in whom there were fewer vaccinations (table $1 \Downarrow$ ). There was a fairly even distribution between sexes in the two cohorts. The number of seizures by calendar time was fairly evenly distributed over the whole period, with a clear dip at the end of 2009/beginning of 2010.

Five people with epilepsy died within the 90 day period after the last vaccination (median age 57.6, SD 24). In all of these people, death occurred more than one month after the seizure. Two people died within a fortnight after vaccination. Two people without epilepsy died within the study period (aged 77 and 85).

\section{Incidence rate of seizures}

We calculated incidence rates of seizures for both cohorts. The incidence rate of epileptic seizures in the cohort with epilepsy was 249 per 1000 person years in the control period before vaccination and slightly lower in the control period after vaccination (229 per 1000 person years) (table $1 \Downarrow)$. In the two risk periods the incidence rate was 258 (day 1-7) and 253 (day 8-30), respectively, per 1000 person years, while it was highest in the buffer period just before vaccination (313 per 1000 person years). The confidence intervals, however, overlapped between periods.

In the cohort without epilepsy the incidence of seizures was considerably lower (range 0.6-1.1 per 1000 person years in the various periods) (table $1 \Downarrow$ ). There was a significant increasing incidence trend over the study period in this cohort $(\mathrm{P}<0.002)$. 


\section{Self controlled case series analysis}

In the main analysis we examined the incidence rate of seizures in the day 1-7 risk period and in the day 8-30 risk period compared with the overall incidence rate in the two control periods before and after vaccination separately for the subcohorts of people with recorded epilepsy and people without recorded epilepsy. For those with epilepsy and seizures there was no increase in risk in the first risk period (day 1-7; relative incidence $1.01,95 \%$ confidence interval 0.74 to 1.39 ) or in the second risk period (day 8-30; $1.00,0.83$ to 1.21 ) (table $2 \Downarrow$ ). For those without epilepsy but with seizures, there was a non-significant decrease in risk in the first risk period $(0.67$, 0.27 to 1.65$)$ and no significant increase in risk in the second risk period $(1.11,0.73$ to 1.70$)$ (table $2 \Downarrow$ ).

We carried several sensitivity analyses to control for robustness of the main analysis. In one sensitivity analysis we included the buffer period (30-1 days before vaccination) in the model. For those with epilepsy and seizures, there was a significant risk increase during the buffer period compared with the control periods (relative incidence 1.33, 95\% confidence interval 1.14 to 1.56 ), while for those without epilepsy but with seizures this was not the case $(1.08,0.71$ to 1.64$)$ (table $2 \Downarrow$ ). Most importantly, however, the relative incidence for any of the risk periods did not change when we included the buffer period in the model, neither for those with or without epilepsy, indicating robust main results.

In a second sensitivity analysis we used only the first control period for comparison with risk periods after vaccination, but took all episodes and doses into account. For those with epilepsy and seizures, the results remained stable compared with the main analysis (table $3 \Downarrow$ ), while for people without epilepsy the risk in the first risk period was equal to unity (relative incidence $0.99,95 \%$ confidence interval 0.39 to 2.53 ), and for the second risk period showed an increased relative incidence of 1.64 , close to significance (95\% confidence interval 0.99 to 2.71 ) (table $3 \Downarrow)$. To further check the robustness of these results we introduced the second control period as a separate parameter into the model, along with the buffer period. The risk estimates in both risk periods remained stable for both subcohorts (table $3 \Downarrow$ ), while the risk estimate for the second control period was non-significantly decreased for those with epilepsy and seizures $(0.89,0.76$ to 1.05$)$, and significantly increased for those without epilepsy but with seizures $(1.88,1.26$ to 2.80$)$. Consequently, a (non-significant) risk increase was also indicated for the buffer period $(1.56,0.95$ to 2.56$)$ for those without epilepsy but with seizures.

We conducted further sensitivity analyses to assess only the first episode of epileptic seizures and the first dose of vaccine. The results from these analyses did not alter the main result of the study, with one exception-namely, in people without epilepsy who received one dose, there was a non-significantly increased risk in the second risk period (relative incidence 1.41, $95 \%$ confidence interval 0.86 to 2.31 ), while the estimate in the first risk period was close to unity $(0.93,0.34$ to 2.53 ) (data not shown).

Additional analyses with the composite outcome of epileptic and febrile seizures confirmed the results shown in tables 2 and 3 .

\section{Discussion}

\section{Principal findings}

This self control case series study did not indicate any increase in the risk of epileptic seizures diagnosed during inpatient or outpatient hospital care after vaccination with a monovalent AS03 adjuvanted pandemic H1N1 influenza vaccine (Pandemrix). The results remained stable in sensitivity analyses, except when we compared the risk periods with the first control period only in people without epilepsy. The estimate of the second control period itself showed a significantly increased risk compared with the first control period, indicating that the incidence of epileptic seizures among the people without epilepsy was almost doubled in the second compared with the first control period. The estimate in the second risk period was also higher in this group, but not significantly so. It is not surprising that the incidence of epilepsy among those without registered epilepsy tended to increase over the study period, as they were free from epileptic seizures and epileptic drugs within at least one year before the pre-vaccination control period. Therefore the incidence of epileptic seizures would be expected to increase over the study period in this group, from a value of zero at the beginning and a peak at the end of the study period, which is what the results reflect.

As the incidence of seizures shortly before vaccination might be artificially lower or higher, we defined a buffer period of 30 days before vaccination (fig $1 \Downarrow$ ). Consistent in all analyses, the buffer period immediately before vaccination indicated an increase in risk for those with epilepsy, a result that was unexpected. A possible reason for this could be that people at risk of epileptic seizures tended to get vaccinated. If these people also get an improvement of their drug treatment to avoid further seizures, however, this could bias the estimates of relative incidence towards a false protective effect. On the other hand, it is not inconceivable that people at risk of epileptic seizures would refrain from vaccination if they were concerned that the vaccine could induce such reactions. Most importantly, in all analyses the estimates of relative incidence in the risk periods remained stable when we included the buffer period in the models.

\section{Relation to other studies}

Epilepsy as an adverse event after vaccination has been reported after use of pertussis vaccination. ${ }^{13}{ }^{14}$ Relevant studies, however, had inconclusive results regarding this association, as summarised in Shorvon and Berg. ${ }^{15}$ The European Medical Agency received notification of seizure with fatal outcome after vaccination with $\mathrm{H} 1 \mathrm{~N} 1$ vaccine in individuals with epilepsy. ${ }^{4}$

Several studies have investigated on the relations between flu vaccines and febrile convulsions and one case report on a 21 year old women. ${ }^{16-19}$ To our knowledge, however, our study is the first population based study on the risk of epileptic convulsion in both adults and children and vaccination against flu that has used healthcare registers instead of a safety monitoring system for identification of adverse advents. ${ }^{20-22}$ Additional analyses of a composite outcome of epileptic and febrile seizures did not alter our main results.

Neurological adverse events were a concern after the H1N1 flu vaccination in 2009 as a sevenfold risk of Guillain-Barré Syndrome during the H1N1 vaccination campaign in 1976 was reported in the United States. ${ }^{5}$ Subsequent studies investigating the risk of Guillain-Barré Syndrome and seasonal flu vaccination could not replicate these results. ${ }^{23}{ }^{24}$ More recently, a multinational case-control study in Europe was conducted to study the association between $\mathrm{H} 1 \mathrm{~N} 1$ vaccination and Guillain-Barré Syndrome, but there was no support for the findings of $1976 .{ }^{25}$ During the H1N1 pandemic, however, there was an increase in incidence of narcolepsy in people vaccinated with Pandemrix in Finland and Sweden. ${ }^{67}$ Considering that 
various neurological disorders have been shown to be associated with both seasonal and pandemic flu vaccinations it is important to investigate every report.

\section{Strength and weaknesses}

The registration of diagnoses in inpatient and outpatient hospital care has been ongoing for many decades and is done routinely in all Swedish counties. The unique Swedish personal identification number has been in use since 1947 and is implemented in all computer aided healthcare systems in Sweden. ${ }^{9}$ The number ensures that each individual can be traced in national computer based registers. As this enables large scale population based studies the results presented here should be valid not only for our study population but most probably for all of Sweden.

Confounding is a common and serious problem in studies of adverse events in people who choose to get vaccinated or not. The commonly used observed and expected analysis is prone to selection bias if the risk of underlying disease is different in people who do and do not get vaccinated. The statistical method we used (SCCS) was specifically developed to investigate adverse events after vaccination. ${ }^{11}$ As the method is based only on cases, and each individual acts as his or her own control, the method controls implicitly for all confounders that do not vary with time over the observation period, such as genetics, location, socioeconomic status, sex, individual frailty, and severity of underlying disease. In our analyses we did not control for age as the observation period was too short for age to realistically influence the results. The SCCS method is designed to investigate outcomes with a rapid onset, which makes it suitable to study epileptic seizures after vaccination as this is an acute event. The method requires that the probability of exposure is not affected by the occurrence of an outcome, which theoretically could be the case for people with epilepsy. This is the reason why we used a cohort of people without recorded epileptic seizures or drugs for epilepsy for comparison. The results for this comparison group were virtually the same as for the epileptic group.

Several limitations also need to be considered. Firstly, we did not investigate the total incidence of epileptic seizures, but rather relevant inpatient and outpatient visits to hospital. Visits at general practitioners were not assessed. A recent Danish validation study found hospital diagnosis of epilepsy to be $81 \%$ correct according to criteria from the International League Against Epilepsy, ${ }^{26}{ }^{27}$ and an earlier study from Stockholm, Sweden, reached a similar result. ${ }^{28}$ As the definition of events rests on diagnoses in inpatient and outpatient care, we could have missed events, both in the control and risk periods. If there was a true increase of epileptic seizures after vaccination, they might not have been captured fully given the limitations in our case definition. The incidence of epileptic seizures might then have been underestimated. On the other hand, the current analysis certainly catches the more severe epileptic seizures leading to inpatient or outpatient visits, and missed diagnoses were probably in less severe affected people. To define the cohorts of people with and without epilepsy, we assessed diagnoses of epilepsy and prescription of antiepileptic drugs during one year before the study period. If the entire medical history of people could have been assessed, there might have been some people in the group without epilepsy who could have been included in the epileptic group. Therefore our defined group of people without epilepsy should be seen as an operational definition, and not necessarily from a medical point of view. Finally, our risk estimates were averages over age and sex and do not allow for differences in risk by age and sex.
Despite these limitations, the results of our analyses were robust, and we could not find any sign of an increase in risk of epileptic seizures after vaccination with Pandemrix among people with epilepsy.

We thank Harald Heijbel (an expert in vaccine safety) at the Swedish Institute for Infectious Disease Control for feedback on the manuscript. We also thank Vincent Guy Bauchan and Dominique Rosillon at GlaxoSmithKline Biologicals for feedback on the statistical analysis and on the draft manuscript.

Contributors: PS, LAD, and JH were responsible for the study design. $\mathrm{LAD}$ collected the data. JH created the database, performed the statistical analysis, and drafted the statistical methods section. LAD drafted the manuscript with PS. CEW performed the statistical analysis together with $\mathrm{JH}$. All authors actively participated in the interpretation of the results and discussed and approved the final version. PS is guarantor.

Funding: This work was supported by a grant from GlaxoSmithKline Biologicals in Belgium. The authors had full access to all the data in the study and had the full responsibility to submit the report for publication. The opinions presented in the report do not necessarily reflect those of the funders.

Competing interests: All authors have completed the ICMJE uniform disclosure form at www.icmje.org/coi_disclosure.pdf (available on request from the corresponding author) and declare that all the authors received unconditional grants from GlaxoSmithKline for the submitted study.

Ethical approval: The study was approved by the ethical review board in Stockholm.

Data sharing: The analysis dataset for this study can be made available to other researchers on request to the authors.

Madsen KM, Hviid A, Vestergaard M, Schendel D, Wohlfahrt J, Thorsen P, et al. A population-based study of measles, mumps, and rubella vaccination and autism. N Engl J Med 2002;347:1477-82.

2 Kaye JA, del Mar Melero-Montes M, Jick $\mathrm{H}$. Mumps, measles, and rubella vaccine and the incidence of autism recorded by general practitioners: a time trend analysis. BMJ 2001;322:460-3

3 Brown NJ, Berkovic SF, Scheffer IE. Vaccination, seizures and 'vaccine damage'. Curr Opin Neurol 2007;20:181-7.

4 European Medicines Agency. Thirteenth pandemic pharmacovigilance update. www.ema. europa.eu/docs/en_GB/document_library/Report/2010/03/WC500075456.pdf.

5 Schonberger LB, Bregman DJ, Sullivan-Bolyai JZ, Keenlyside RA, Ziegler DW, Retailliau $\mathrm{HF}$, et al. Guillain-Barre syndrome following vaccination in the National Influenza Immunization Program, United States, 1976-1977. Am J Epidemiol 1979;110:105-23.

6 Nohynek H, Jokinen J, Partinen M, Vaarala O, Kirjavainen T, Sundman J, et al. AS03 adjuvanted $A H 1 \mathrm{~N} 1$ vaccine associated with an abrupt increase in the incidence of childhood narcolepsy in Finland. PloS One 2012;7:e33536.

$7 \quad$ MPA. Occurrence of narcolepsy with cataplexy among children and adolescents in relation to the H1N1 pandemic and Pandemrix vaccinations. Secondary Occurrence of narcolepsy with cataplexy among children and adolescents in relation to the H1N1 pandemic and Pandemrix vaccinations 2011. www.lakemedelsverket.se/upload/nyheter/2011/ Fallinventeringsrapport_pandermrix_110630.pdf.

8 Epilepsy Foundation America. Secondary Epilepsy Foundation America 2010. www. epilepsyfoundation.org.

9 Ludvigsson JF, Otterblad-Olausson P, Pettersson BU, Ekbom A. The Swedish personal identity number: possibilities and pitfalls in healthcare and medical research. Eur $J$ Epidemiol 2009;24:659-67.

10 Ulm K. A simple method to calculate the confidence interval of a standardized mortality ratio (SMR). Am J Epidemiol 1990;131:373-5.

11 Farrington $\mathrm{CP}$, Nash J, Miller E. Case series analysis of adverse reactions to vaccines: a comparative evaluation. Am J Epidemiol 1996;143:1165-73.

12 Whitaker HJ, Farrington CP, Spiessens B, Musonda P. Tutorial in biostatistics: the self-controlled case series method. Stat Med 2006;25:1768-97.

13 Kulenkampff M, Schwartzman JS, Wilson J. Neurological complications of pertussis inoculation. Arch Dis Child 1974;49:46-9.

14 Gale JL, Thapa PB, Wassilak SG, Bobo JK, Mendelman PM, Foy HM. Risk of serious acute neurological illness after immunization with diphtheria-tetanus-pertussis vaccine. A population-based case-control study. JAMA 1994;271:37-41.

15 Shorvon S, Berg A. Pertussis vaccination and epilepsy -an erratic history, new research and the mismatch between science and social policy. Epilepsia 2008;49:219-25.

16 Leroy Z, Broder K, Menschik D, Shimabukuro T, Martin D. Febrile seizures after 2010-2011 influenza vaccine in young children, United States: a vaccine safety signal from the vaccine adverse event reporting system. Vaccine 2012;30:2020-3.

17 Mitrakrishnan S, Ranjanie G, Thirunavakarasu T, Manjula C, Nayananjani K. Seizures and retrograde amnesia with cerebrospinal fluid changes following $\mathrm{H} 1 \mathrm{~N} 1$ influenza vaccination. Vaccine 2011;29:6369-70.

18 Polkinghorne BG, Muscatello DJ, Macintyre CR, Lawrence GL, Middleton PM, Torvaldsen $\mathrm{S}$. Relationship between the population incidence of febrile convulsions in young children 


\section{What is already known on this topic}

Vaccination against A/H1N1 PDM09 has previously been associated with an increased risk of neurological events such as Guillain-Barré syndrome and is possibly associated with an increased risk of narcolepsy

Epileptic seizure have also been reported after vaccination against pertussis

\section{What this study adds}

Vaccination against A/H1N1 PDM09 does not seem to be a risk factor for epileptic seizures in people with or without a previous diagnosis of epilepsy

in Sydney, Australia and seasonal epidemics of influenza and respiratory syncytial virus, 2003-2010: a time series analysis. BMC Infect Dis 2011;11:291.

19 Stowe J, Andrews N, Bryan P, Seabroke S, Miller E. Risk of convulsions in children after monovalent H1N1 (2009) and trivalent influenza vaccines: a database study. Vaccine 2011;29:9467-72.

20 Munch JS, Johnsen BH, Birkeland I, Finne M, Utkilen T, Boe T, et al. A study of side-effects of Pandemrix influenza ( $\mathrm{H} 1 \mathrm{~N} 1)$ vaccine on board a Norwegian Naval vessel. Int Marit Health 2010;62:246-50

21 Mayet A, Ligier C, Gache K, Manet G, Nivoix P, Dia A, et al. Adverse events following pandemic influenza vaccine Pandemrix reported in the French military forces-2009-2010. Vaccine 2011;29:2576-81.

22 Van Puijenbroek EP, Broos N, van Grootheest K. Monitoring adverse events of the vaccination campaign against influenza A (H1N1) in the Netherlands. Drug Saf 2010;33:1097-108

23 Lasky T, Terracciano GJ, Magder L, Koski CL, Ballesteros M, Nash D, et al. The Guillain-Barre syndrome and the 1992-1993 and 1993-1994 influenza vaccines. N Engl J Med 1998;339:1797-802.

24 Juurlink DN, Stukel TA, Kwong J, Kopp A, McGeer A, Upshur RE, et al. Guillain-Barre syndrome after influenza vaccination in adults: a population-based study. Arch Intern Med 2006;166:2217-21.
25 Dieleman J, Romio S, Johansen K, Weibel D, Bonhoeffer J, Sturkenboom M. Guillain-Barre syndrome and adjuvanted pandemic influenza A (H1N1) 2009 vaccine: multinational case-control study in Europe. BMJ 2011;343:d3908.

26 Christensen J, Vestergaard M, Olsen J, Sidenius P. Validation of epilepsy diagnoses in the Danish National Hospital Register. Epilepsy Res 2007;75:162-70.

27 Guidelines for epidemiologic studies on epilepsy. Commission on Epidemiology and Prognosis, International League Against Epilepsy. Epilepsia 1993;34:592-6. 28 Tomson T. Mortality in epilepsy. J Neurol 2000;247:15-21.

Accepted: 02 November 2012

Cite this as: BMJ 2012;345:e7594

This is an open-access article distributed under the terms of the Creative Commons Attribution Non-commercial License, which permits use, distribution, and reproduction in any medium, provided the original work is properly cited, the use is non commercial and is otherwise in compliance with the license. See: http://creativecommons.org/licenses/bync/2.0/ and http://creativecommons.org/licenses/by-nc/2.0/legalcode. 


\section{Tables}

Table 1 | Frequency and incidence rates of seizures per 1000 person years among vaccinated population with and without previous epileptic seizures, by sex, age group, and study period

\begin{tabular}{|c|c|c|c|c|c|c|}
\hline & \multicolumn{3}{|c|}{ People with epilepsy } & \multicolumn{3}{|c|}{ People without epilepsy } \\
\hline & No & Seizures (No of people) & Incidence rate $(95 \% \mathrm{Cl})$ & No & Seizures (No of people) & Incidence rate $(95 \% \mathrm{Cl})$ \\
\hline Total & 7787 & $987(738)$ & 253 (238 to 270 ) & 365611 & $168(121)$ & 0.89 (0.76 to 1.03$)$ \\
\hline \multicolumn{7}{|l|}{ Sex: } \\
\hline Male & 3455 & $487(361)$ & 282 (258 to 308 ) & 173112 & $80(58)$ & $0.89(0.70$ to 1.11$)$ \\
\hline Female & 4332 & $500(377)$ & 231 (211 to 252) & 192499 & $88(63)$ & 0.88 (0.71 to 1.09 ) \\
\hline \multicolumn{7}{|c|}{ Age (years): } \\
\hline $0-24$ & 776 & $340(241)$ & 809 (725 to 900$)$ & 116409 & $72(46)$ & 1.08 (0.85 to 1.37$)$ \\
\hline $25-49$ & 2227 & 264 (203) & 239 (211 to 270$)$ & 99774 & $36(25)$ & $0.73(0.51$ to 1.01$)$ \\
\hline $50-74$ & 3445 & $293(230)$ & 171 (152 to 192$)$ & 115339 & $34(25)$ & 0.60 (0.41 to 0.83$)$ \\
\hline$\geq 75$ & 1339 & $90(64)$ & 137 (110 to 168$)$ & 34089 & $26(25)$ & 1.55 (1.01 to 2.27 ) \\
\hline \multicolumn{7}{|c|}{ Period (days around vaccination): } \\
\hline$-90--31$ & 7787 & $318(294)$ & 249 (222 to 277 ) & 365611 & $36(34)$ & 0.60 (0.42 to 0.83$)$ \\
\hline$-30--1$ & 7787 & $200(187)$ & 313 (271 to 359$)$ & 365611 & $28(25)$ & 0.93 (0.62 to 1.35$)$ \\
\hline $1-7$ & 7787 & $41(40)$ & 258 (185 to 350 ) & 365611 & $5(5)$ & $0.62(0.20$ to 1.45$)$ \\
\hline 8-30 & 7785 & $131(121)$ & 253 (212 to 300$)$ & 365551 & $27(27)$ & 1.03 (0.68 to 1.49$)$ \\
\hline $31-90$ & 7769 & $297(258)$ & 229 (204 to 256 ) & 365231 & $72(60)$ & $1.11(0.87$ to 1.40$)$ \\
\hline
\end{tabular}


Table 2| Relative incidence of seizures in risk periods compared with control period (day 90-31 before vaccination and day 31-90 after vaccination) for people with epilepsy and with seizures $(n=738)$ and for people without epilepsy but with seizures ( $n=121)$ for all episodes, all doses, and inpatient and outpatient seizures

\begin{tabular}{|c|c|c|c|c|}
\hline & \multicolumn{2}{|c|}{ With epilepsy, with seizures $(n=738$ ) } & \multicolumn{2}{|c|}{ Without epilepsy, with seizures $(n=121)$} \\
\hline & No of seizures & Relative incidence $(95 \% \mathrm{Cl})$ & No of seizures & Relative incidence $(95 \% \mathrm{Cl})$ \\
\hline \multicolumn{5}{|l|}{ Main model } \\
\hline \multicolumn{5}{|l|}{ Risk period: } \\
\hline Day 1-7 & 41 & $1.01(0.74$ to 1.39$)$ & 5 & $0.67(0.27$ to 1.65$)$ \\
\hline Day 8-30 & 131 & $1.00(0.83$ to 1.21$)$ & 27 & $1.11(0.73$ to 1.70$)$ \\
\hline \multicolumn{5}{|c|}{ Model including buffer period (day $30-1$ before vaccination) } \\
\hline Buffer period & 200 & $1.33(1.13$ to 1.56$)$ & 28 & $1.08(0.71$ to 1.64$)$ \\
\hline \multicolumn{5}{|l|}{ Risk period: } \\
\hline Day 1-7 & 41 & $0.96(0.69$ to 1.33$)$ & 5 & $0.67(0.27$ to 1.63$)$ \\
\hline Day $8-30$ & 131 & $0.95(0.77$ to 1.16$)$ & 27 & $1.10(0.72$ to 1.68$)$ \\
\hline
\end{tabular}


Table 3| Relative incidence of seizures in risk periods compared with first control period (day 90-31 before vaccination) for people with epilepsy and with seizures $(n=738)$ and for people without epilepsy but with seizures $(n=121)$ for all episodes, all doses, and inpatient and outpatient seizures

\begin{tabular}{|c|c|c|c|c|}
\hline & \multicolumn{2}{|c|}{ With epilepsy and seizures $(n=738)$} & \multicolumn{2}{|c|}{ Without epilepsy, with seizures $(n=121)$} \\
\hline & No of seizures & Relative incidence $(95 \% \mathrm{Cl})$ & No of seizures & Relative incidence $(95 \% \mathrm{Cl})$ \\
\hline \multicolumn{5}{|l|}{ Main model } \\
\hline \multicolumn{5}{|l|}{ Risk period: } \\
\hline Day 1-7 & 41 & 0.95 (0.69 to 1.32$)$ & 5 & 0.99 (0.39 to 2.53 ) \\
\hline Day 8-30 & 131 & 0.94 (0.77 to 1.16$)$ & 27 & 1.64 (0.99 to 2.71$)$ \\
\hline \multicolumn{5}{|c|}{ Model including buffer period ( $30-1$ days before vaccination) } \\
\hline Buffer period & 200 & $1.26(1.06$ to 1.50$)$ & 28 & 1.56 (0.95 to 2.56$)$ \\
\hline \multicolumn{5}{|l|}{ Risk period: } \\
\hline Day $1-7$ & 41 & 0.96 (0.69 to 1.33$)$ & 5 & $0.97(0.38$ to 2.47$)$ \\
\hline Day 8-30 & 131 & $0.95(0.77$ to 1.16$)$ & 27 & $1.61(0.97$ to 2.65$)$ \\
\hline \multicolumn{5}{|c|}{ Control period: } \\
\hline Day 31-90 & 297 & 0.89 (0.76 to 1.05$)$ & 72 & $1.88(1.26$ to 2.80$)$ \\
\hline
\end{tabular}




\section{Figures}

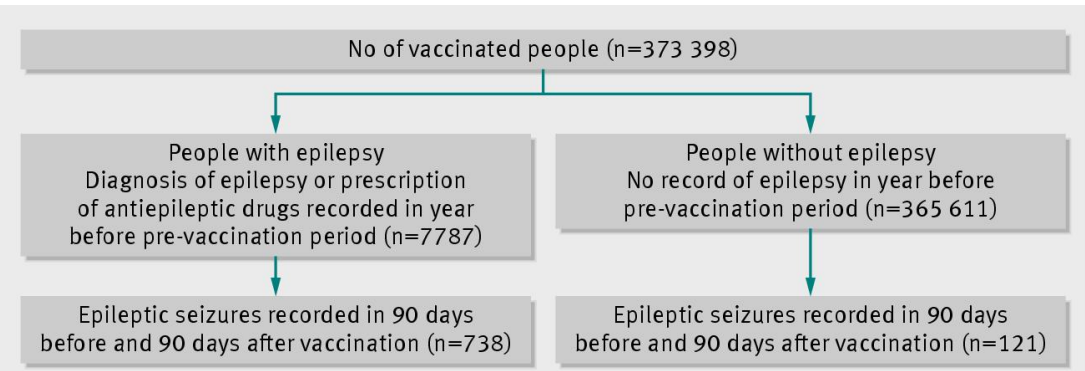

Fig 1 Schematic description of study cohorts from people who were vaccinated against pandemic H1N1 2009 flu

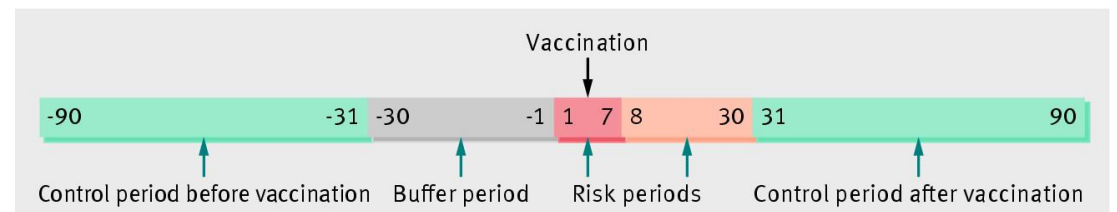

Fig 2 Risk periods after vaccination and control periods before and after vaccination. Numbers represent days from vaccination (day 1 ) 